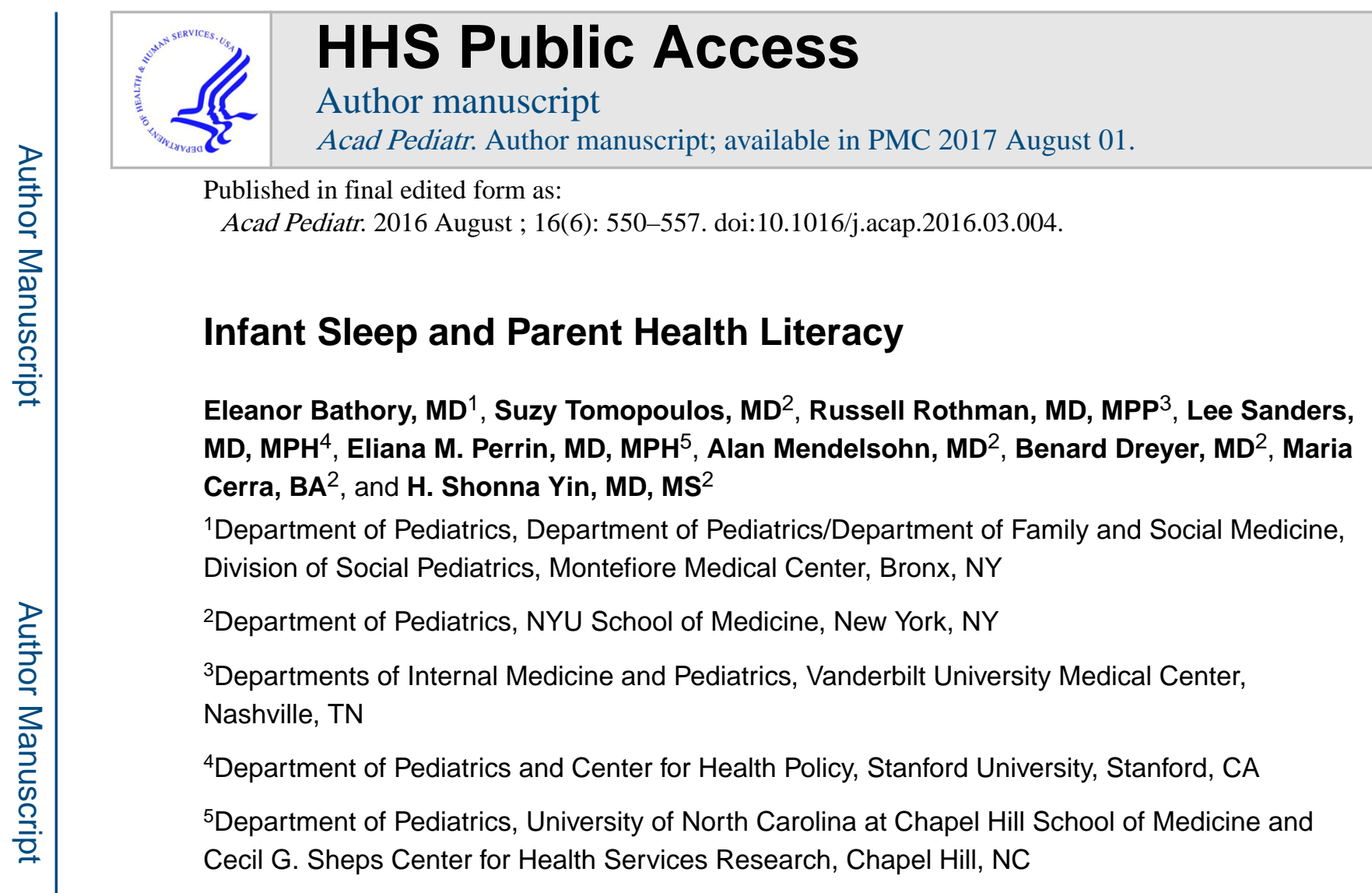

\title{
Abstract
}

Objective-Child sleep problems are prevalent and have been linked to poor behavior, worse school performance, and obesity. Low health literacy (HL) is associated with suboptimal parenting practices and worse health outcomes, but the relationship between parent HL and child sleeprelated issues is not known. We examined the association between parent HL and child sleeprelated issues.

Corresponding author: Eleanor Bathory, MD, ebathory@montefiore.org; Department of Pediatrics/Department of Family and Social Medicine, Division of Social Pediatrics, Montefiore Medical Center, 3455 Jerome Avenue, Bronx, New York.

Conflicts of Interest: The authors have no conflicts of interest to disclose.

Previous Presentation: Findings from this study was presented in part at the Pediatric Academic Societies Annual Meeting; 4/28/13; Boston, MA.

\section{ADDITIONAL CONTRIBUTIONS}

We thank co-Investigator Anna Maria Patino Fernandez, $\mathrm{PhD}$, as well as research coordinators Andrea J. Bacchus, BA, Evelyn Cruzatte, BA, Joanne Finkle, JD, RN, Beth Throop, BA, Sophie Ravanbakht, BA, Lucila Bloise, BA, and Daniela Quesada, BA, MPH, and our entire research staff, for their assistance.

\section{AUTHOR CONTRIBUTIONS}

Drs. Yin and Bathory had full access to all of the data in the study and take responsibility for the integrity of the data and the accuracy of the data analysis.

Study concept and design: Bathory, Yin, Tomopoulos, Rothman, Sanders, Perrin, Dreyer, Mendelsohn.

Acquisition of data: Yin, Rothman, Sanders, Perrin, Cerra.

Analysis and interpretation of data: Bathory, Yin, Rothman, Sanders, Perrin, Mendelsohn, Dreyer, Cerra.

Drafting of the manuscript: Bathory, Yin.

Critical revision of the manuscript for important intellectual content: Bathory, Yin, Tomopoulos, Rothman, Sanders, Perrin, Mendelsohn, Dreyer, Cerra.

Obtained funding: Yin, Rothman, Sanders, Perrin.

Administrative, technical, or material support: Yin, Tomopoulos, Cerra.

Study supervision: Yin, Rothman, Sanders, Perrin, Mendelsohn, Dreyer.

Publisher's Disclaimer: This is a PDF file of an unedited manuscript that has been accepted for publication. As a service to our customers we are providing this early version of the manuscript. The manuscript will undergo copyediting, typesetting, and review of the resulting proof before it is published in its final citable form. Please note that during the production process errors may be discovered which could affect the content, and all legal disclaimers that apply to the journal pertain. 
Design/Methods-Cross-sectional analysis of data from caregivers enrolled in a cluster randomized trial of a primary care-based child obesity prevention program in 4 pediatric clinics. Parent HL assessed using the Short Test of Functional HL in Adults. At the 9-month well-child visit, sleep-related factors were assessed: presence of TV in room where child sleeps, regular naptimes and bedtimes ( $>5$ days/week), low daytime and nighttime sleep duration ( $>1$ SD below mean based on national data). Adjusted logistic regression analyses performed.

Results-557 caregivers of 9-month olds enrolled (49.7\% Hispanic, 26.9\% Black, 56.2\% < $\$ 20 \mathrm{~K}$ annual income); $49.6 \%$ reported having a TV in the room where their child sleeps; $26.6 \%$ did not have regular naptimes/bedtimes. Median (IQR) sleep duration was 2.3(1.5-3.0) hours (daytime), 9.0(8.0-10.0) hours (night) (30.2\% low daytime; $20.3 \%$ low night sleep duration). Children of parents with low HL were more likely to have a bedroom TV (66.7 v. $47.7 \%, \mathrm{p}=0.01$;

$\mathrm{AOR}=2.2[95 \% \mathrm{CI}: 1.1-4.3]$ ) and low nighttime sleep (37.0 v. $18.5 \%, \mathrm{p}=0.002$; $\mathrm{AOR}=2.4[1.2-4.8])$.

Conclusions-Low parent HL is associated with TV in the bedroom and low night sleep duration. Additional study is needed to further explore these associations and intervention strategies to address child sleep problems.

\section{Keywords}

health literacy; infants; television; sleep; sleep hygiene; sleep duration

\section{INTRODUCTION}

One in four parents of infants and toddlers report a concern about sleep, with common problems involving sleep regularity and duration. ${ }^{1,2}$ Child sleep problems have been linked to issues with attention, behavior and school functioning, and can have negative secondary effects on maternal and family well-being. ${ }^{1}$ Irregular sleep schedules have been shown to alter sleep-wake cycles, negatively impact sleep quality, and lead to sleep problems. ${ }^{1-5}$ Parenting practices related to sleep in infancy likely contribute to the development of child sleep issues. ${ }^{2}$

Parents can help children establish healthy sleep habits that are sustained through childhood. ${ }^{1,6}$ The AAP Bright Futures program recommends family behaviors that promote good sleep hygiene through the creation of a calm and sleep-promoting environment, including absence of a TV in the room where the child sleeps and consistent routines, such as maintenance of consistent bedtimes and regular nap times. ${ }^{7}$

Parent health literacy is one factor that may contribute to suboptimal child sleep-related parenting practices and the development of child sleep issues. Low parent health literacy has been linked to poor parenting practices, including suboptimal child feeding practices (eg. lower breastfeeding rates, pressuring feeding styles), ${ }^{8}$ as well as poor child health outcomes (eg. worse asthma-related morbidity). ${ }^{9,10}$ Individuals with low health literacy have less access to high quality health information and greater difficulty understanding and acting on health recommendations ${ }^{9,10}$ Parents, particularly pregnant and new mothers, rely on obstetric and pediatric providers, as well as parenting books and online sources, for information on common parenting questions, especially with respect to child sleep. ${ }^{11}$ In the 
context of sleep problems, parents with low health literacy may have decreased access to information on how to promote healthy sleep practices and thus may be less likely to access and incorporate health recommendations made by groups such as the AAP/Bright Futures regarding both sleep hygiene and television viewing. ${ }^{7,12}$

To date, there has been limited study of the relationship between parent health literacy and child sleep. In this study, we therefore sought to examine the association between parent health literacy and specific aspects of child sleep, in particular, presence of TV in the room where the child sleeps, sleep regularity, and sleep duration.

\section{METHODS}

\section{Study Design}

This was a cross-sectional analysis of self-reported data from caregivers of infants participating in the Greenlight study, a cluster randomized controlled trial of a primary care based, low literacy health communication intervention to reduce obesogenic behaviors in early childhood. Greenlight study details have been previously described. ${ }^{13}$

Enrollment of consecutive eligible parent-child dyads was performed across four academic medical centers: NYU School of Medicine/Bellevue Hospital Center, Vanderbilt University School of Medicine, University of North Carolina School of Medicine at Chapel Hill, and University of Miami Miller School of Medicine. NYU and Vanderbilt were randomized to receive the obesity prevention intervention and UNC and Miami were active controls. The low literacy Greenlight toolkits were available for use as part of counseling for well-child visits from 2-months through 2 years of age. Toolkits included low literacy booklets focused on evidence-based obesity prevention messages targeting each well-child visit, along with supplemental booklets that providers had the option to give. The 4-page "Sleep Time for your Baby and Toddler" supplement focuses on sleep routines, eliminating overnight feeding after 6 months of age, and avoidance of bottle propping and cereal in the bottle. The active control sites received an injury prevention program based on AAP The Injury Prevention Program (TIPP), with no emphasis on low literacy communication, but did include one sleep-related message - putting the infant to sleep on his back.

Inclusion criteria for parent-child dyads in the Greenlight study were: child ( 6 to < 16 weeks) presenting for a 2-month well-child visit with a trained pediatric resident, with an English or Spanish-speaking caregiver who plans to return to clinic for all well-child visits through child's 2 year check-up. Exclusion criteria were: child's birth <34 weeks' gestational age or birth weight $<1500$ grams, 2 -month weight/length $<3^{\text {rd }}$ percentile, diagnosis of failure to thrive or known medical problems that may affect the ability to gain weight; and parent $<18$ years-old or with significant mental or neurological illness, or poor visual acuity (Figure 1). Written and verbal informed consent was obtained from all caregivers. Institutional Review Board approval was obtained from each participating site.

\section{Data Obtained}

Data was collected via parent interview at the 2 and 9-month well-child visits by trained research assistants in English or Spanish (caregiver preference). REDCap (Research 
Electronic Data Capture) tools hosted at Vanderbilt University was used to manage study data. ${ }^{14}$

Dependent Variables: Sleep-related factors-Child sleep data was assessed by caregiver report at the 9-month well-child visit, and included presence of a TV in the room where the child sleeps, sleep regularity, and sleep duration. The 9-month well-child visit was chosen because most 9-month old infants are physiologically capable of sleeping through the night without feeding, daytime naps are likely to be distinct from nighttime sleep, and regular meal times are being established, all making this an important developmental point for establishing and maintaining clear sleep routines. ${ }^{4}$ All sleep-related survey questions were adapted from surveys used in similar populations. ${ }^{3,15-17}$

Presence of TV in the room where child sleeps: Presence of a TV in the room where the child sleeps was assessed using the question: "Is there a working TV in the room where your child sleeps?" (yes/ no.) ${ }^{18}$

Sleep regularity: Sleep regularity refers to the frequency with which caregivers reported that their infant goes to sleep at about the same time every day. Naptime regularity was assessed with the question: "Does your child usually take a nap at about the same time each day?" Parents who answered yes were asked: "In the past 7 days, how many days did your child go to sleep at his/her regular nap time?" Bedtime regularity was assessed with the question: "Does your child usually go to bed at about the same time every night?" Parents who answered yes were asked: "In the past 7 days, how many days did your child go to sleep at his/her regular bedtime?" Naptimes and bedtimes were considered regular if the caregivers reported consistency for $\geq 5$ days per week. Overall child sleep regularity was categorized as: neither regular naptime nor bedtime, regular naptime, regular bedtime, and both regular naptime and bedtime. ${ }^{3,6}$

Sleep duration: Sleep duration was assessed by determining the number of hours the infant slept during the day and night. Daytime duration was assessed using the question: "For how many hours does your baby usually nap in the morning or afternoon?" and nighttime sleep duration was assessed using the question "For how many hours does your baby usually sleep during the night?"15

Low sleep duration was defined as greater than 1 standard deviation (SD) below the mean for 8-10 month old infants, based on recent data from the National Sleep Foundation. ${ }^{19} \mathrm{We}$ used a cut off based on nationally representative data to strengthen the generalizability of study findings; 1 SD below the mean was used rather than a 2 SD cut-off, which would have categorized only those infants in the bottom $2.5 \%$ as having low sleep duration. Thus, low daytime sleep duration was defined as $<1.8$ hours/day and low nighttime sleep duration was defined as < 7.5 hours/night; sleep duration results were dichotomized as "low" or "adequate."

Primary Independent Variable: Health Literacy-Parent health literacy, the primary independent variable, was collected at the 2-month well-child visit using the Short Test of Functional Health Literacy in Adults (STOFHLA) ${ }^{20}$ The STOFHLA, validated in English 
and Spanish, is one of the most common assessments used for health literacy research. ${ }^{10}$ Parents were categorized as having inadequate (score 0-16), marginal (17-22), or adequate (23-36) health literacy. For analyses, the inadequate and marginal health literacy groups were combined into one "low literacy" category, as in prior studies. ${ }^{21,22}$

Sociodemographic Variables-Sociodemographic characteristics collected by caregiver report at the 2-month well-child visit included child's date of birth, gender, and out of home child care (any vs. none) and caregiver's age, race/ethnicity (Hispanic, White non-Hispanic, Black non-Hispanic, and Other non-Hispanic), language (English or Spanish), education (less than high school vs. high school graduate or higher), depressive symptoms (evaluated with the Center for Epidemiologic Studies Depression Scale (>16 indicates clinically significant levels of psychological distress $)),{ }^{23}$ household annual income $(<\$ 10,000$, $\$ 10,000-19,000, \$ 20,000-40,000,>\$ 40,000$, don't know), number of adults ( $\geq 18$ years) in household ( 1 vs. $\geq 2$ adults) and number of children in household ( 1 vs. $\geq 2$ children).

Statistical Analysis-Unadjusted associations between parent health literacy and child sleep hygiene variables were performed using Mann-Whitney U (continuous variables; sleep duration not normally distributed) and chi square analysis (categorical variables). Multiple logistic regression analyses were performed to assess associations between parent health literacy and child sleep-related issues (presence of TV in room where child sleeps, sleep regularity, sleep duration), with a priori inclusion of: child age at 9-month well-child visit, gender, out of home care; parent age, race/ethnicity, language, education, and depressive symptoms; household income, \# of adults in household, \# of children in household; study site. Analyses were also conducted excluding infants born at $<37$ weeks gestation, given differences in physiology and parent-child relationships for these preterm babies which may impact on child sleep issues. Data were analyzed using SPSS software, with a 2-tailed pvalue $<0.05$ considered statistically significant.

\section{RESULTS}

Between April 28, 2010 and August 30, 2012, 865 families were consecutively enrolled in the Greenlight study at their child's 2-month check-up. Details of cohort enrollment have previously been reported. ${ }^{24} 613$ returned for 9-month well-child visit assessments, of which 3 did not complete health literacy assessments and 32 did not complete sleep assessment questions. 21 were excluded as they reported daily sleep durations of $<4$ hours, and were considered outliers. There was no statistically significant difference in health literacy levels between outliers and those included in the analysis. Table 1 describes the 557 parent-child dyads that were included in this analysis. Parents had a mean (SD) STOFHLA score of $31.7(7.4) .54$ parents $(9.7 \%)$ were categorized as low health literacy $(6.5 \%$ inadequate and $3.2 \%$ marginal).

\section{Child Sleep}

Child sleep-related issues were common (Table 2). Nearly half of parents reported that their infants slept in a room that contained a working TV (49.6\%), and one-quarter reported neither regular nap nor bedtimes $(26.6 \%)$. In a 24-hour period, 9-month old infants in our 
sample slept a median (interquartile range (IQR)) of 2.3(1.5-3.0) hours during the daytime, 9.0(8.0-10.0) hours during the nighttime, and 11.3(9.5-13.0) hours total in a day. In our sample, $30.2 \%$ of infants were categorized as having a low daytime sleep duration $(<1.8$ hours) and $20.3 \%$ low nighttime sleep duration ( $<7.5$ hours) (Table 2$)$. No difference in presence of child sleep-related issues was found by intervention status.

\section{Association between Parent Health Literacy and Child Sleep-related Issues}

Compared to parents with adequate health literacy, parents with low health literacy were more likely to have TV's in the room where their infants slept (66.7\% v $47.7 \%, \mathrm{p}=0.01)$. In adjusted analyses (Table 3), parents with low health literacy had twice the odds of reporting a TV in the room where their infant slept compared to those with adequate health literacy (AOR 2.2, 95\% CI: 1.1-4.3). There was no significant difference between the number of children without nap or bedtime sleep regularity by parent health literacy level in unadjusted and adjusted analyses (Table 3).

Infants of parents with low health literacy had decreased nighttime sleep duration compared to infants of parents with adequate health literacy (median (IQR) 8.0(6.0-10.0) vs. 9.0(8.010.0) hours), $\mathrm{p}=0.01$ ). Infants of parents with low health literacy were also more likely to be categorized as having low infant nighttime sleep duration (37.0\% v 18.5\%, $\mathrm{p}=0.002)$. In adjusted analyses (Table 3), parents with low health literacy had over twice the odds of having an infant with low nighttime sleep duration (AOR 2.4, 95\% CI: 1.2-4.8).

\section{DISCUSSION}

This study is the first to look at associations between parent health literacy and child sleeprelated issues among infants in families with low SES backgrounds. We found high rates of sleep-related problems. Half of parents reported that their infants had a TV in the room where they slept; more than a quarter reported irregular nap and bed times; and over a fifth reported low daytime or nighttime sleep duration. Interestingly, compared to parents with adequate health literacy, parents with low health literacy had more than twice the odds of reporting a TV in the room where their infant slept and lower infant nighttime sleep duration. Importantly, the relationship between health literacy and these sleep-related issues remained significant after adjusting for socioeconomic status and other confounders.

Presence of a TV in the room where the child sleeps is recognized to have a strong effect on sleep: children with TV in their bedroom get less sleep at night, go to bed later at night, and watch an average of $>4$ more hours of TV per week, compared to those without a TV in the bedroom. ${ }^{3,6}$ Despite AAP recommendations that discourage children under the age of 2 from watching any TV, studies consistently show that young children watch excessive amounts of TV starting in infancy. ${ }^{3,12,25}$ Recent large studies in the US show approximately $20 \%$ of infants have a TV in the room in which they sleep compared to half of parents of 9month olds in our sample. ${ }^{26}$ While our rate was substantially higher than most studies, few studies have examined the issue of TV in the bedroom among low income minority populations; one study of families with similar demographic characteristics did find similar rates. $^{27}$ 
We found that presence of a TV in the bedroom where the child slept was more common among parents with low health literacy. Though, to date, there has been limited study of the association between parent health literacy and TV use among infants or young children, there is a documented relationship between increased child TV use and lower parent education and income levels, parent characteristics both often associated with low parent health literacy. ${ }^{28}$ Given the prevalence of bedroom TVs in our sample, and the previously documented negative associations, our findings point to the need to target families with low health literacy as part of educational interventions related to reducing bedroom TVs.

Promotion of consistent bedtimes and naptimes is another parenting practice critical to healthy sleep and is thought to improve synchronization of sleep-wake cycle and circadian rhythms. ${ }^{5}$ Children with regular sleep times, tend to fall asleep more easily and therefore get both more and better sleep. Consistent sleep times, like other daily routines, are calming, comforting, and even empowering for young children by providing predictability 1,2,6 Irregular sleep times, particularly with respect to bedtimes, are associated with more daytime sleepiness and behavior problems. ${ }^{4,16}$ In our sample, less than half of parents reported both regular nap and bedtimes. While our data shows similar rates of naptime regularity as other studies, our rate of bedtime regularity was lower. ${ }^{3,16}$ We found no significant correlation between sleep regularity and health literacy, but the relatively low percentage of families with regular sleep times, particularly bedtimes, suggests that education regarding the importance of sleep regularity to child well-being and sleep quality may be warranted across literacy groups.

In addition to deficiencies in sleep regularity, our study found substantially shorter daily sleep duration, with 11 hours total per 24-hours, compared to the 14-15 hours per day recommended by the CDC. Similarly, our population reported shorter nighttime sleep durations than that reported in the literature: 8.6 vs. 9.3-11.2 hours/night. ${ }^{6,29-31}$ Our finding that low parental health literacy was associated with significantly shorter nighttime sleep duration has not been previously documented. However, shorter nighttime sleep duration in families with low health literacy is consistent with the previously observed relationship between parental education and sleep duration in school age children. ${ }^{32}$

While our data clearly shows an association between low health literacy and specific aspects of child sleep issues, we do not know the reason for this correlation. In general, parents with low health literacy have more difficulty understanding, accessing, and gauging the reliability of public health messages and information from health care professionals, however it is unclear why some behaviors are linked to low health literacy while others are not. ${ }^{9,10,32}$ Further study is needed to clarify how parent health literacy influences specific child sleep practices and behaviors in order to develop effective interventions to address child sleeprelated issues. While screening for family health literacy is likely to be impractical in pediatrician's offices, knowledge of local population data regarding health literacy may be helpful. Use of low literacy verbal and written communication strategies, such as "teachback" and use of plain language written materials, may be especially helpful in promoting improved infant sleep for families with low health literacy, but would likely benefit families across literacy levels. ${ }^{9,10}$ 
Critical to implementation of any sleep intervention is recognition that pediatricians receive little formal training in medical school or in residency regarding sleep medicine, do not adequately address sleep problems in routine well-child visits, and underdiagnose sleep issues. ${ }^{33,34}$ Thus, there is a widespread need for interventions regarding sleep, for both health care providers and the families they care for.

It is important to note that issues of sleep, including TV presence in the bedroom, may be influenced by social determinants of health including poverty. In low literacy, low SES populations there are likely to be many social issues such as housing insecurity, crowding, food insecurity, job insecurity, under and unemployment and tenuous childcare. Thus interventions targeting sleep problems should account for these issues, in order to successfully promote optimal sleep practices.

This study has several limitations. This is a secondary data analysis of cross-sectional data and thus causal inference is limited. The scope and depth of the questions regarding child sleep is limited: we did not assess specific bedtimes and wake times, bedtime routines, cosleeping status, number or length of nighttime awakening; the quantity, content, or timing of bedroom TV use; bedroom sharing; or specific child health or behavioral issues which may have contributed to sleep problems. Sleep data was collected at 9-months, when most infants are able to sleep through the night and are establishing routines; however we recognize that at 9-months, infants also develop separation anxiety and new gross motor skills that can result in new sleep disruptions. Future study of sleep issues across time points would be helpful to explore these issues. While we included demographic confounders in adjusted analyses, we could not specifically look at how close families were to the federal poverty line; instead, we included in our adjusted models annual household income as a categorical variable, along with number of adults and children in the home. We relied on parent report which is subject to social desirability and recall bias. Although surveys were administered by trained research assistants, who assisted parents with any questions that arose during survey completion, there may have been measurement error associated with the mathematical calculation required to determine sleep duration. Data on naptime sleep may also be less reliable for families whose children attend daycare or other childcare settings. Sleep questions were modified from other research tools, but not from a validated instrument. Information on specific anticipatory guidance topics discussed by individual providers was not collected as part of the study. Counseling related to sleep, however, was not considered to be a key component of either the Greenlight or TIPP intervention, and we did not find differences in child sleep-related issues by intervention status. Additionally, while the STOFHLA is one of the most commonly used research tools for assessing health literacy; we found a relatively small percentage of parents in our diverse and low income sample with low health literacy. Other studies have had similar findings when studying a young adult population and it has been suggested that existing cut-points may underestimate the number of low health literate participants in this group. ${ }^{35}$ We believe, however, that these cut-points remain useful, as those who are categorized as having inadequate or marginal health literacy using the STOFHLA have children who are likely to be at particular risk for worse health outcomes. Further research is needed to further investigate whether different cut-points for the STOFHLA should be used for this younger adult population, or whether it may be better to use other health literacy measures for this population. There may be 
residual confounding variables that have not been accounted for in our analysis. Finally, there was a time gap between assessments of health literacy and sleep: parent health literacy was assessed when infants were 2-months old and sleep-related issues when infants were 9months old. We do not, however, expect that parent performance on health literacy assessments would have changed significantly during this interval.

In summary, many families, especially parents with low health literacy, experience child sleep-related problems. The high prevalence, as well as the recognized medical, psychological and social impact of child sleep issues, suggests that increased provider awareness of sleep-related issues is needed, along with the development of targeted interventions to assist providers and families to more effectively manage child sleep problems, especially for those children whose parents have low health literacy.

\section{Acknowledgments}

Funding:

This research was supported with funding from the National Institutes of Health (NIH) / National Institute of Child Health and Human Development (NICHD) (R01 HD049794), NIH / National Center for Advancing Translational Sciences (NCATS) (U54 RR023499, UL1RR025747, UL1 TR000038), and the KiDS of NYU Foundation. Dr. Yin is currently supported by HRSA (12-191-1077- Academic Administrative Units in Primary Care).

\section{References}

1. Mindell JA, Owens JA, Carskadon MA. Developmental features of sleep. Child Adolesc Psychiatr Clin N Am. Oct; 1999 8(4):695-725. [PubMed: 10553199]

2. Davis KF, Parker KP, Montgomery GL. Sleep in infants and young children: part two: common sleep problems. Journal of Pediatric Health Care. 2004; 18(3):130-137. [PubMed: 15129213]

3. Thompson DA, Christakis DA. The association between television viewing and irregular sleep schedules among children less than 3 years of age. Pediatrics. 2005; 116(4):851-856. [PubMed: 16199693]

4. Mindell, JA.; Owens, JA. A clinical guide to pediatric sleep: diagnosis and management of sleep problems. Lippincott Williams \& Wilkins; 2009.

5. Ferber R. Circadian rhythm sleep disorders in childhood. Principles and practice of sleep medicine in the child. 1995:91-105.

6. Mindell JA, Meltzer LJ, Carskadon MA, Chervin RD. Developmental aspects of sleep hygiene: findings from the 2004 National Sleep Foundation Sleep in America Poll. Sleep medicine. Aug; 2009 10(7):771-779. [PubMed: 19285450]

7. Hagan, JF.; Shaw, JS.; Duncan, PM. Bright futures: Guidelines for health supervision of infants, children, and adolescents. American Academy of Pediatrics; Elk Grove Village, IL: 2008.

8. Fredrickson D, Washington R, Pham N, Jackson T, Wiltshire J, Jecha L. Reading grade levels and health behaviors of parents at child clinics. Kansas medicine: the journal of the Kansas Medical Society. 1995; 96(3):127. [PubMed: 8583738]

9. DeWalt DA, Hink A. Health literacy and child health outcomes: a systematic review of the literature. Pediatrics. Nov; 2009 124(Suppl 3):S265-274. [PubMed: 19861480]

10. Berkman ND, Sheridan SL, Donahue KE, et al. Health literacy interventions and outcomes: an updated systematic review. Evidence report/technology assessment. Mar.2011 (199):1-941. [PubMed: 23126607]

11. Bernhardt JM, Felter EM. Online pediatric information seeking among mothers of young children: results from a qualitative study using focus groups. Journal of medical Internet research. 2004; $6(1)$ 
12. Brown A. Media use by children younger than 2 years. Pediatrics. Nov; 2011 128(5):1040-1045. [PubMed: 22007002]

13. Sanders LM, Perrin EM, Yin HS, Bronaugh A, Rothman RL. "Greenlight study": a controlled trial of low-literacy, early childhood obesity prevention. Pediatrics. 2014; 133(6):e1724-e1737. [PubMed: 24819570]

14. Harris PA, Taylor R, Thielke R, Payne J, Gonzalez N, Conde JG. Research electronic data capture (REDCap)-A metadata-driven methodology and workflow process for providing translational research informatics support. Journal of biomedical informatics. 2009; 42(2):377. [PubMed: 18929686]

15. Taveras EM, Rifas-Shiman SL, Oken E, Gunderson EP, Gillman MW. Short sleep duration in infancy and risk of childhood overweight. Archives of pediatrics \& adolescent medicine. Apr; 2008 162(4):305-311. [PubMed: 18391138]

16. Hale L, Berger LM, LeBourgeois MK, Brooks-Gunn J. Social and demographic predictors of preschoolers' bedtime routines. J Dev Behav Pediatr. Oct; 2009 30(5):394-402. [PubMed: 19745760]

17. [Accessed September 11, 2015] Fragile Families and Child Wellbeing Study. 2013. http:// www.fragilefamilies.princeton.edu/documentation.asp

18. Woodard, EH.; Gridina, N. [Accessed September 11, 2015] Media in the home. The Fifth Annual Survey of Parents And Children. 2000. http://cdn.annenbergpublicpolicycenter.org/wp-content/ uploads/survey72.pdf

19. [Accessed September 11, 2015] Sleep in America Poll. 2004. http://sleepfoundation.org/sleeppolls-data/sleep-in-america-poll/2004-children-and-sleep

20. Baker DW, Williams MV, Parker RM, Gazmararian JA, Nurss J. Development of a brief test to measure functional health literacy. Patient Educ Couns. Sep; 1999 38(1):33-42. [PubMed: 14528569]

21. Guerra CE, Dominguez F, Shea JA. Literacy and knowledge, attitudes, and behavior about colorectal cancer screening. Journal of health communication. 2005; 10(7):651-663. [PubMed: $16278201]$

22. Hironaka LK, Paasche-Orlow MK, Young RL, Bauchner H, Geltman PL. Caregiver health literacy and adherence to a daily multi-vitamin with iron regimen in infants. Patient education and counseling. 2009; 75(3):376-380. [PubMed: 19395227]

23. Radloff LS. The CES-D scale A self-report depression scale for research in the general population. Applied psychological measurement. 1977; 1(3):385-401.

24. Yin, Hsiang Shonna, MLMS, MD, MPH; Rothman, Russell L., MD, MPP; Shustak, Rachel, BSKE, MS; Shintani, Ayumi, PhD, MPH; Cerra, Maria E., BA; Cruzatte, Evelyn F, BEMP, MD, MPH. Parent Health Literacy and "Obesogenic" Feeding and Physical Activity-related Infant Care Behaviors. Journal of Pediatrics. Under review.

25. Perrin EM, Rothman RL, Sanders LM, et al. Racial and Ethnic Differences Associated With Feeding-and Activity-Related Behaviors in Infants. Pediatrics. 2014; 133(4):e857-e867. [PubMed: 24639273]

26. Stanger, J.; Jamieson, K. Television in the home 1998: The third annual national survey of parents and children. Philadelphia: Annenberg Public Policy Center; 1998.

27. Dennison BA, Erb TA, Jenkins PL. Television viewing and television in bedroom associated with overweight risk among low-income preschool children. Pediatrics. Jun; 2002 109(6):1028-1035. [PubMed: 12042539]

28. Roberts DF. Kids \& Media@ the New Millennium: A Kaiser Family Foundation Report. A Comprehensive National Analysis of Children's Media Use. Executive Summary. 1999

29. Iglowstein I, Jenni OG, Molinari L, Largo RH. Sleep duration from infancy to adolescence: reference values and generational trends. Pediatrics. Feb; 2003 111(2):302-307. [PubMed: 12563055]

30. Sadeh A, Mindell JA, Luedtke K, Wiegand B. Sleep and sleep ecology in the first 3 years: a webbased study. Journal of sleep research. Mar; 2009 18(1):60-73. [PubMed: 19021850]

31. Williams JA, Zimmerman FJ, Bell JF. Norms and trends of sleep time among US children and adolescents. JAMA pediatrics. Jan; 2013 167(1):55-60. [PubMed: 23403646] 
32. Buckhalt JA, El-Sheikh M, Keller PS, Kelly RJ. Concurrent and longitudinal relations between children's sleep and cognitive functioning: the moderating role of parent education. Child development. May-Jun;2009 80(3):875-892. [PubMed: 19489909]

33. Owens JA. The practice of pediatric sleep medicine: results of a community survey. Pediatrics. 2001; 108(3):e51-e51. [PubMed: 11533369]

34. Meltzer LJ, Johnson C, Crosette J, Ramos M, Mindell JA. Prevalence of diagnosed sleep disorders in pediatric primary care practices. Pediatrics. Jun; 2010 125(6):e1410-1418. [PubMed: 20457689]

35. Kumar D, Sanders L, Perrin EM, et al. Parental understanding of infant health information: health literacy, numeracy, and the Parental Health Literacy Activities Test (PHLAT). Academic pediatrics. 2010; 10(5):309-316. [PubMed: 20674532] 


\section{What's New}

Suboptimal parenting practices related to infant sleep are common. Presence of TV in the child's bedroom and low child night time sleep duration are associated with low parent health literacy; targeted intervention may be beneficial for these at-risk parents. 


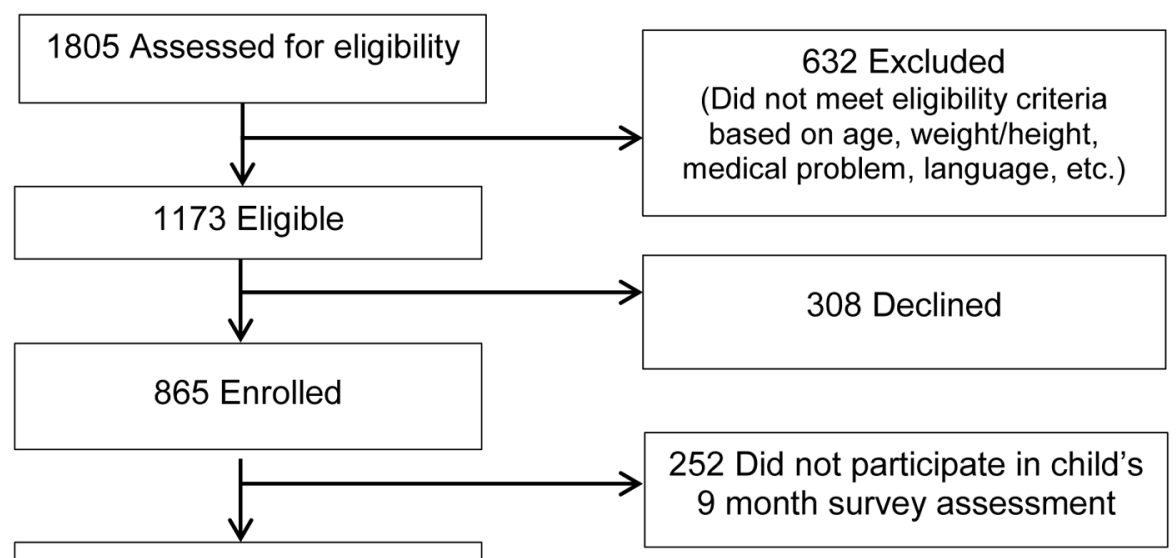

613 Participated in child's 9 month survey assessment

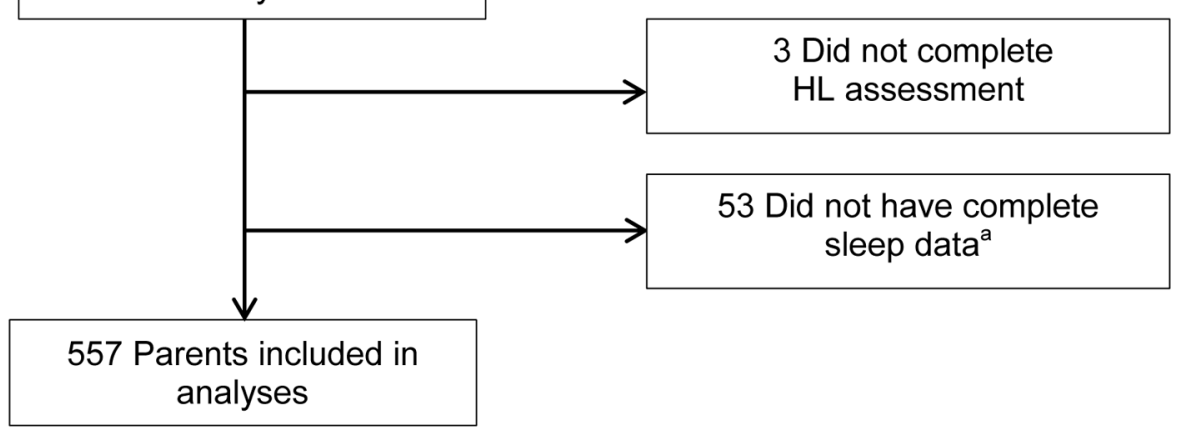

FIGURE 1.

Study enrollment

ancludes 21 who had implausible sleep duration of $<4$ hours, as these were considered outliers. 
TABLE 1

Characteristics of study population ${ }^{a}$

\begin{tabular}{|c|c|}
\hline Child Characteristics & Mean (SD) or n (\%) \\
\hline Child Age, months & $9.5(0.7)$ \\
\hline \multicolumn{2}{|l|}{ Child Gender } \\
\hline Male & $267(47.9)$ \\
\hline Female & $290(52.1)$ \\
\hline Child Out of Home Care: Any & $48(8.6)$ \\
\hline Child Health Insurance coverage: Medicaid / none & $496(89.0)$ \\
\hline \multicolumn{2}{|l|}{ Parent Characteristics } \\
\hline Parent Age, years $b$ & $27.8(5.8)$ \\
\hline \multicolumn{2}{|l|}{ Relationship to Child } \\
\hline Mother & $530(95.2)$ \\
\hline Father & $25(4.5)$ \\
\hline Other & $2(0.4)$ \\
\hline Parent Non-US Born & $290(52.1)$ \\
\hline \multicolumn{2}{|l|}{ Parent Race/Ethnicity } \\
\hline Hispanic & $277(49.7)$ \\
\hline \multicolumn{2}{|l|}{ Non-Hispanic } \\
\hline White, non-Hispanic & $104(18.7)$ \\
\hline Black, non-Hispanic & $150(26.9)$ \\
\hline Other, non-Hispanic & $26(4.7)$ \\
\hline Parent Language, Spanish ${ }^{c}$ & $204(36.6)$ \\
\hline Parent Education, Less than HS Graduate & $146(26.2)$ \\
\hline Parent Health Literacy score ${ }^{d}$ & $31.7(7.4)$ \\
\hline \multicolumn{2}{|l|}{ Parent Health Literacy ${ }^{d}$} \\
\hline Inadequate Literacy & $36(6.5)$ \\
\hline Marginal Literacy & $18(3.2)$ \\
\hline Adequate Literacy & $503(90.3)$ \\
\hline Parent Depressive Symptoms $e$ & $85(15.3)$ \\
\hline \multicolumn{2}{|l|}{ Household Characteristics } \\
\hline \multicolumn{2}{|l|}{ Income (annual) } \\
\hline$<\$ 10,000$ & $150(26.9)$ \\
\hline
\end{tabular}




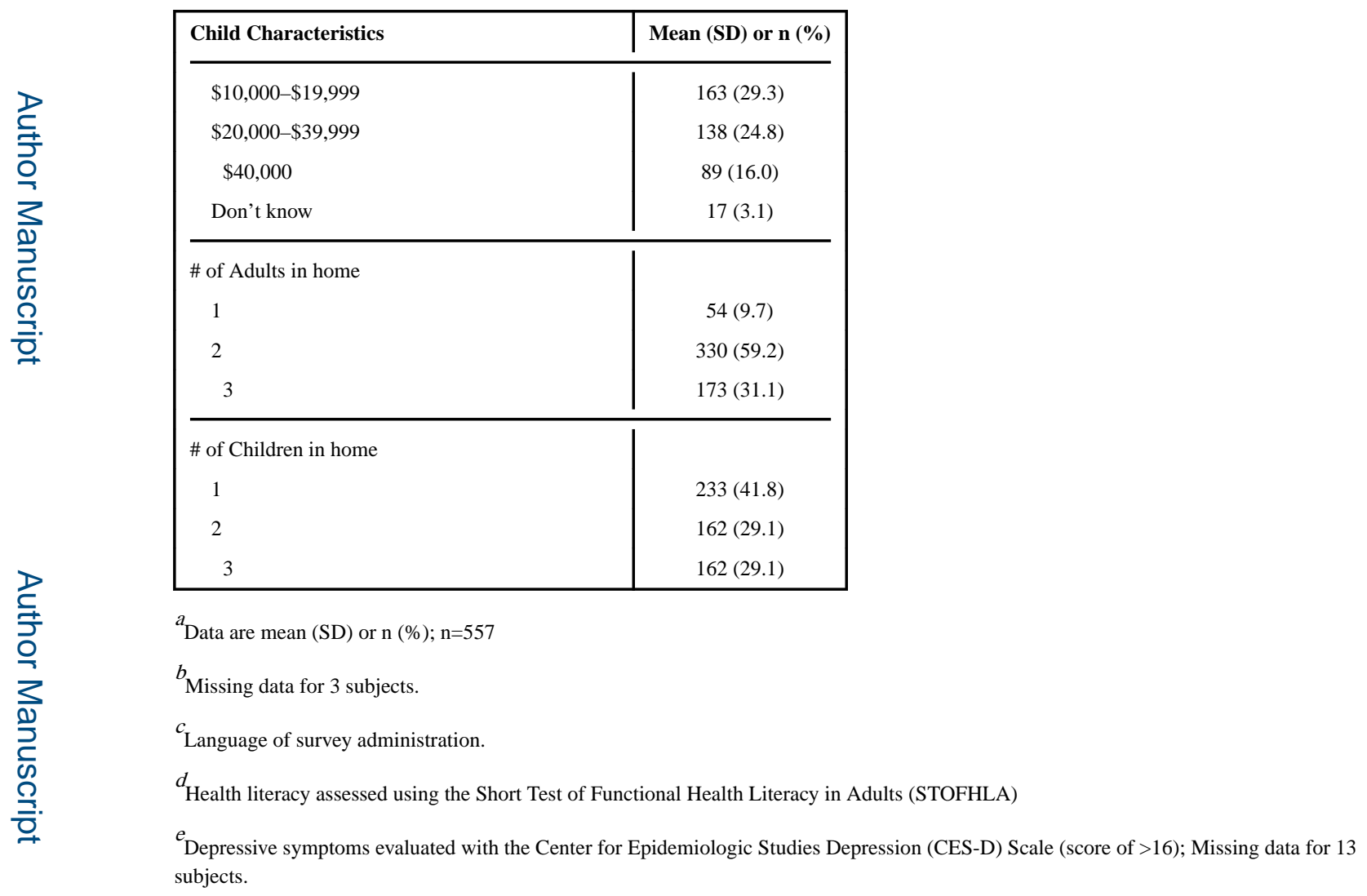




\section{TABLE 2}

Descriptive data for sleep-related variables

\begin{tabular}{|l|c|}
\hline & $\mathbf{N}(\%)$ \\
\hline TV in room where child sleeps & \\
Yes & $276(49.6)$ \\
No & \\
\hline Sleep regularity ${ }^{a}$ & $140.4)$ \\
Neither nap nor bed & $45(8.1)$ \\
Nap only & $114(20.5)$ \\
Bed only & $250(44.9)$ \\
Both nap and bed & \\
\hline Daily sleep duration, median $(\mathrm{IQR}), \mathrm{h}$ & $2.3(1.5-3.0)$ \\
Day & $9.0(8.0-10.0)$ \\
Night & $11.3(9.5-13.0)$ \\
Total & $168(30.2)$ \\
\hline Low day sleep duration $(<1.8 \mathrm{~h})^{b}$ & $113(20.3)$ \\
\hline Low night sleep duration $(<7.5 \mathrm{~h})^{c}$ & \\
\hline
\end{tabular}

${ }^{a}$ Sleep times were considered regular if parent reported consistent sleep time 25 days per week

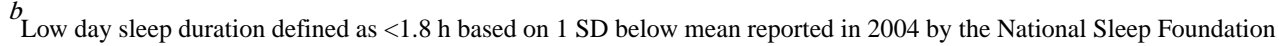

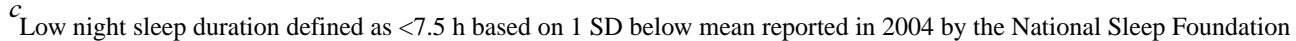




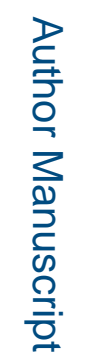

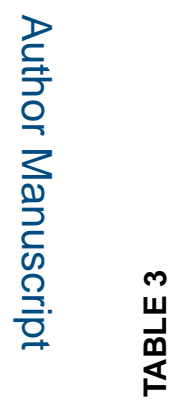

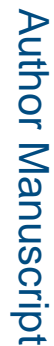

을

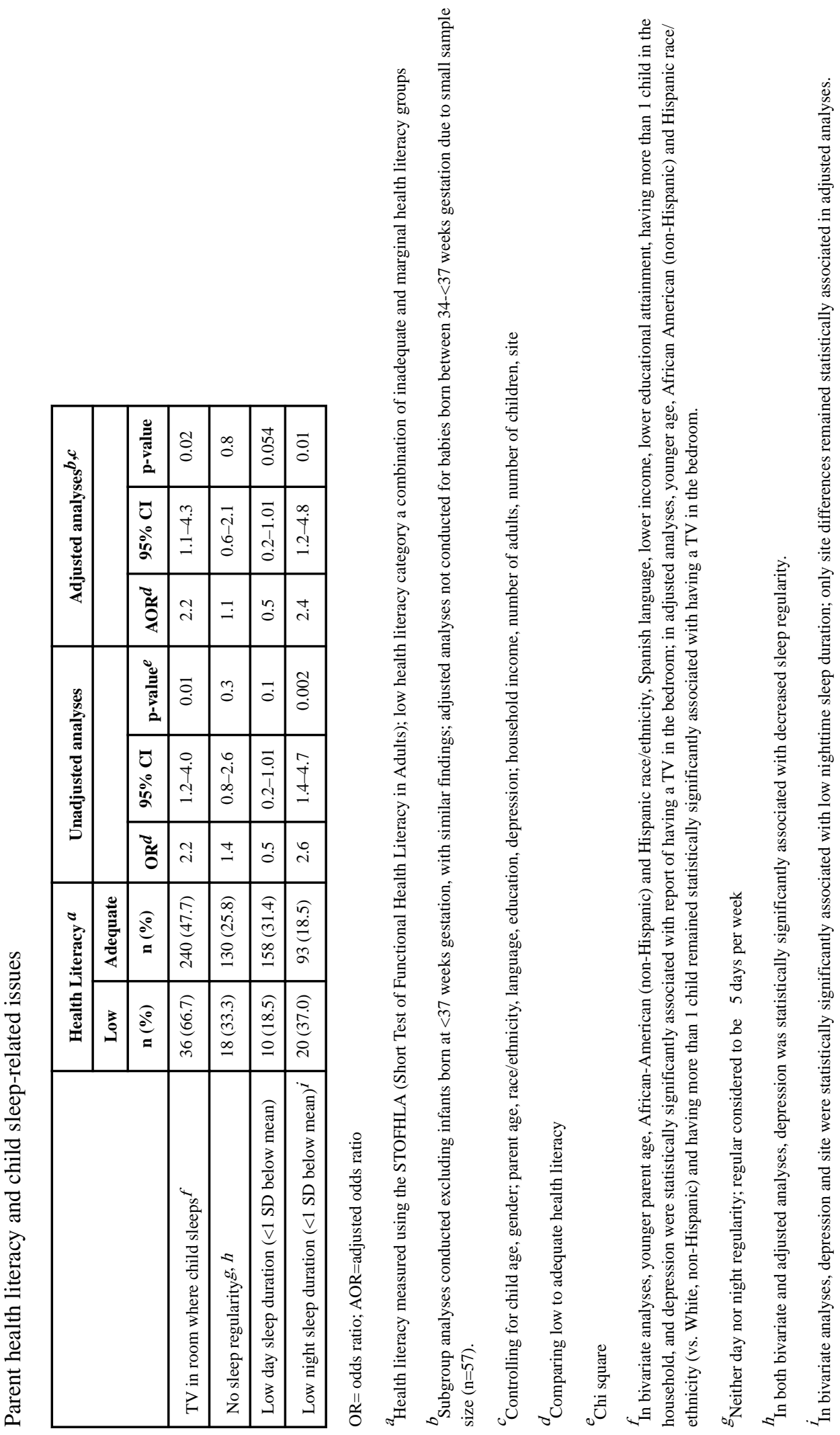

Acad Pediatr. Author manuscript; available in PMC 2017 August 01. 